\title{
On averaging for switched linear differential algebraic equations
}

\author{
Luigi Iannelli $i^{\natural}$, Carmen Pedicini ${ }^{\natural}$, Stephan Trenn $\diamond$, Francesco Vasca ${ }^{\natural}$
}

\begin{abstract}
Averaging is an effective technique which allows the analysis and control design of nonsmooth switched systems through the use of corresponding simpler smooth averaged systems. Approximation results and stability analysis have been presented in the literature for dynamic systems described by switched ordinary differential equations. In this paper the averaging technique is shown to be useful also for the analysis of switched systems whose modes are represented by means of differential algebraic equations (DAEs). An approximation result is derived for a simple but representative homogenous switched DAE with periodic switching signals and two modes. Simulations based on a simple electrical circuit model illustrate the theoretical result.
\end{abstract}

\section{INTRODUCTION}

Averaging theory is a powerful tool to analyze nonlinear systems and it has been proved that trajectories of the averaged system are close to those of the unaveraged system and an relationship between the stability properties of the two systems has been established [1]. In the last few years averaging theory for switched systems has attracted a growing interest in the control literature where different approaches and points of view related to the switched system characteristics are considered: non-periodic switching functions [2] [3], pulse modulations [4], dithering [5], [6], effects of exogenous inputs [7], hybrid systems framework [8]. The paper [9] presents an overview on the averaging results for switched systems which switches among modes each represented by means of possibly nonlinear ordinary differential equations. Averaging of fast switching systems is also an effective technique used in many engineering applications [10].

The representation of switched systems by means of switching ordinary differential equations (ODEs) might limit the class of systems which can be considered. For instance, a switched system characterized by modes with different algebraic constraints, which may imply state jumps at the switching time instants, cannot be represented by means of switched ODEs. In this case one can use a representation through switched differential algebraic equations (DAEs). A switched (linear) DAE is a system consisting of a family of linear DAEs and a policy that at each time instant selects the active subsystem among a set of possible modes. The selection policy is usually described by means of a switching function, which is a function of time. In this paper we consider homogeneous linear switched DAEs of the form [11]

$$
E_{\sigma} \dot{x}=A_{\sigma} x
$$

(দ) Department of Engineering, University of Sannio, 82100 Benevento, Italy, email: \{carmen.pedicini, francesco.vasca, luigi.iannelli\} @unisannio.it

$(\diamond)$ Department of Mathematics, University of Kaiserslautern, 67663 Kaiserslautern, Germany, email: trennemathematik.uni-kl.de where the switching function $\sigma(t):[0, \infty) \rightarrow \Sigma$ is a piecewise constant function right-continuous, that selects at each time instant $t$ the index of the active mode from the finite index set $\Sigma=\{1,2, \ldots, M\}$. We restrict our attention here mainly on the case $M=2$ (c.f. Remark 4) and on periodic switching signals. Each mode is given by the matrices $E_{i}, A_{i} \in \mathbb{R}^{n \times n}$ and it is assumed that the matrix pair $\left(E_{i}, A_{i}\right)$ is regular, i.e. the polynomial $\operatorname{det}\left(s E_{i}-A_{i}\right)$ is not the zero polynomial, for each $i \in \Sigma$.

Switched DAEs occur, for example, in modeling electrical circuits with switches or when modeling possible faults in systems where each (faulty and non-faulty) configuration is described by a DAE [12], [13]. In spite of the presence of possible state jumps, the state evolution in switched DAEs might exhibit an average (smooth) emergent behavior. In that sense our scope is to give some theoretical justifications for averaging applications for this class of nonsmooth systems by presenting a new averaging theorem based on direct comparison of the solutions of the original and the proposed averaged systems. Although we concentrate on a specific class of switched (linear) DAEs, at the best of our knowledge this is the first time in the literature that the averaging technique is applied to switched DAEs.

The paper is organized as follows. Section II recalls some preliminary results. In particular the classical averaging approach for ODE is presented as well as a short review of the solution theory for switched DAEs is given. Section III discusses the averaging approach for switched DAEs and we first show via a simple example that in general a averaged model does not exist. However, if we make the assumption that the consistency projectors commute then we can show that an averaged model exists and the solutions of the switched DAE converge to the averaged solution for increasing switching frequency. In Section IV an example based on a simple electrical circuit model is presented which illustrates our theoretical results. Section V concludes the paper by proposing some future research directions and the major open issues.

\section{Preliminaries}

For readability of the paper we first introduce some basic definitions and a lemma.

Definition 1 (Big O notation): Consider two functions $f, g:(0, \infty) \rightarrow \mathcal{V}$, where $\mathcal{V}$ is some normed vector space with norm $\|\cdot\|$. We write

$$
f(x)=O(g(x)) \text { for } x \rightarrow 0
$$

if and only if there exist constants $K$ and $\bar{x}>0$ such that

$$
\|f(x)\| \leqslant K\|g(x)\| \text { for all } 0<x \leqslant \bar{x} .
$$


Lemma 1: Consider two commuting matrices $M_{1}$ and $M_{2}$, with $M_{1}$ such that $M_{1}^{2}=M_{1}$. Then the following holds

Proof:

$$
\operatorname{im} M_{1} M_{2}=\operatorname{im} M_{1} \cap \operatorname{im} M_{2} .
$$

"ᄃ" Clearly, it holds that

$$
\operatorname{im} M_{1} M_{2} \subseteq \operatorname{im} M_{1},
$$

and by using that $M_{1}$ and $M_{2}$ are commuting matrices we also have

$$
\operatorname{im} M_{1} M_{2}=\operatorname{im} M_{2} M_{1} \subseteq \operatorname{im} M_{2} .
$$

This shows $\operatorname{im} M_{1} M_{2} \subseteq \operatorname{im} M_{1} \cap \operatorname{im} M_{2}$.

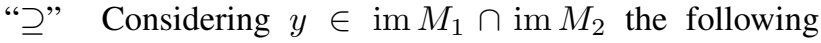
holds:

$$
\begin{aligned}
y \in & \operatorname{im} M_{1} \cap \operatorname{im} M_{2} \\
\Leftrightarrow & \exists x_{1} \in \mathbb{R}^{n}, \exists x_{2} \in \mathbb{R}^{n}: \\
& M_{1} x_{1}=M_{2} x_{2}=y \\
\Rightarrow & y \doteq M_{1}^{2} x_{1} \stackrel{\star}{=} M_{1} M_{2} x_{2} \\
\Rightarrow & \exists x \in \mathbb{R}^{n}: y=M_{1} M_{2} x \\
\Leftrightarrow & y \in \operatorname{im} M_{1} M_{2},
\end{aligned}
$$

where for $\bullet$ we used $M_{1}^{2}=M_{1}$ and for $\star$ we used $M_{1} x_{1}=M_{2} x_{2}$. This concludes the proof.

\section{A. The Averaging approach for switched ODEs}

Consider the homogeneous switched linear system

$$
\dot{x}=A_{\sigma} x,
$$

and assume that the switching function $\sigma$ is periodic of period $p$. If the period is sufficiently small the averaged model can be introduced. The averaged system approximates the behavior of the system (5) and can be represented as

$$
\dot{x}_{\mathrm{av}}=A_{\mathrm{av}} x_{\mathrm{av}},
$$

where $A_{\mathrm{av}}$ is a constant matrix defined as

$$
A_{\mathrm{av}}=\frac{1}{p} \int_{0}^{p} A_{\sigma(s)} \mathrm{d} s=\sum_{i=1}^{M} \frac{\tau_{i}}{p} A_{i},
$$

where $\tau_{i}$ is the total time duration of the mode $\sigma(t)=i$ for $t \in[0, p]$ and $d_{i}=\tau_{i} / p$ is the duty cycle of the $i$ th mode.

By using the arguments shown in [14] and [5] it is possible to show the following approximation result.

Theorem 1: Consider the switched systems (5) and the corresponding averaged system (6), (7), with initial conditions $x(0)=x_{0}$ and $x_{\mathrm{av}}(0)=x_{0}$. Assume that the switched signal $\sigma$ is periodic of period $p$, then for any given $\bar{t}$ and for any initial condition $x_{0} \in \mathbb{R}^{n}$ the following holds

$$
\left\|x(t)-x_{\mathrm{av}}(t)\right\|=O(p), \quad \forall t \in[0, \bar{t}] .
$$

The purpose of this paper is the generalization of this result to switched DAEs with two modes.

\section{B. The quasi-Weierstrass form and consistency projectors}

Consider a non-switched DAE

$$
E \dot{x}=A x
$$

with $E$ and $A \in \mathbb{R}^{n \times n}$ and differentiable solutions $x: \mathbb{R} \rightarrow$ $\mathbb{R}^{n}$. When the matrix $E$ is invertible, (9) reduces to a more familiar ordinary differential equation, so in the following the matrix $E$ is in general singular. However, we assume that the matrix pair $(E, A)$ is a regular, i.e. $\operatorname{det}(s E-A)$ is not the zero polynomial. It is well known that this is the case if, and only if, there exist invertible transformation matrices $S, T \in \mathbb{R}^{n \times n}$ that put the matrices in the quasi Weierstrass form [15]

$$
(S E T, S A T)=\left(\left[\begin{array}{cc}
I & 0 \\
0 & N
\end{array}\right],\left[\begin{array}{ll}
J & 0 \\
0 & I
\end{array}\right]\right)
$$

where $N \in \mathbb{R}^{n_{2} \times n_{2}}$,with $0 \leqslant n_{2} \leqslant n$, is a nilpotent matrix and $J \in \mathbb{R}^{n_{1} \times n_{1}}$, with $n_{1}=n-n_{2}$, is some matrix and $I$ is the identity matrix of appropriate size. Following [16] we call (10) quasi Weierstrass form as we do not assume that $J$ and $N$ are in Jordan canonical form. In [16] it was shown that the transformation matrix can be represented as $T=[V ; W]$ and $S=[E V ; A W]^{-1}$ with $\operatorname{im} V=\mathcal{V}^{*}$ and $\operatorname{im} W=\mathcal{W}^{*}$ where $\mathcal{V}^{*}$ and $\mathcal{W}^{*}$ are obtained through the Wong sequences [17], given by

$$
\begin{gathered}
\mathcal{V}_{0}:=\mathbb{R}^{n}, \mathcal{V}_{i+1}:=A^{-1}\left(E \mathcal{V}_{i}\right), i \in \mathbb{N}, \mathcal{V}^{*}:=\bigcap_{i \in \mathbb{N}} \mathcal{V}_{i} \\
\mathcal{W}_{0}:=\{0\}, \mathcal{W}_{i+1}:=A^{-1}\left(E \mathcal{W}_{i}\right), i \in \mathbb{N}, \mathcal{W}^{*}:=\bigcup_{i \in \mathbb{N}} \mathcal{W}_{i}
\end{gathered}
$$

It is easy to see that a DAE in Weierstrass form consists of two independent parts: an "ODE part" given by

$$
\dot{y}=J y
$$

and a "pure DAE part" given by

$$
N \dot{z}=z,
$$

where the pure DAE part only has the solution $z=0$. Hence the classical solutions of a regular $\operatorname{DAE}(E, A)$ are given by the ODE (11) and the coordinate transformation

$$
x=T\left(\begin{array}{l}
y \\
0
\end{array}\right) .
$$

This leads to the definition of the so called consistency projectors. The consistency projector $\Pi$ of the matrices pair $(E, A)$ is defined as

$$
\Pi=T\left[\begin{array}{ll}
I & 0 \\
0 & 0
\end{array}\right] T^{-1},
$$

where the block sizes correspond to the block size in the quasi Weierstrass form (10). The consistency projector characterizes the space within all solutions of (9) evolve, i.e. the consistency space is im $\Pi$; furthermore it plays a role when considering inconsistent initial values as they occur 
when switching between different DAEs. To describe the DAE solution it is possible to introduce the flow matrix

$$
A^{\text {diff }}=T\left[\begin{array}{ll}
J & 0 \\
0 & 0
\end{array}\right] T^{-1} .
$$

Note that, due to the special structure of the consistency projector $\Pi$ and $A^{\text {diff }}$,

$$
A^{\mathrm{diff}} \Pi=A^{\mathrm{diff}}=\Pi A^{\mathrm{diff}} .
$$

By using the flow matrix it is possible [18] to introduce an ODE system

$$
\dot{x}=A^{\text {diff }} x,
$$

and show that each solution of (9) also solves (17).

\section{Solutions of switched DAEs}

Consider the switched DAE (1). To ensure the uniqueness of solutions we assume that each matrix pair $\left(E_{i}, A_{i}\right)$ is regular, and (e.g. by calculating the corresponding Wong sequences) we assume knowledge of the quasi-Weierstrass form (10) with corresponding transformation matrices $T_{i}, S_{i}$, consistency projectors $\Pi_{i}$ and flow matrices $A_{i}^{\text {diff }}$. Moreover we assume impulse-free solutions for any switching signal, which can be characterized [12] by the condition

$$
E_{j}\left(I-\Pi_{j}\right) \Pi_{i}=0, \forall i, j \in\{1,2, \ldots, M\} .
$$

Any solution of each individual DAE $E_{i} \dot{x}=A_{i} x$, evolves within the consistency space and at a switching time $t_{k}$, a continuous extension to the future does not exist in general, because the value $x\left(t_{k}-\right):=\lim _{\varepsilon \searrow 0} x(t-\varepsilon)$ need not be within the consistency space corresponding to the DAE after the switch. Therefore it is necessary to allow for solutions with jumps. Indeed, it can be shown [12] that the jump from an inconsistent to a consistent initial value is uniquely given by the consistency projector $\Pi_{i_{k}}$ corresponding to the system $\left(E_{i_{k}}, A_{i_{k}}\right)$ activated at the switching time $t_{k}$ :

$$
x\left(t_{k}\right)=\Pi_{i_{k}} x\left(t_{k-1}-\right) .
$$

Hence, invoking (17), the solution $x$ on the interval $\left[t_{k}, t_{k+1}\right)$ is given by

$$
\begin{aligned}
x(t) & =e^{A_{i_{k}}^{\mathrm{diff}}\left(t-t_{k}\right)} x\left(t_{k}\right) \\
& =e^{A_{i_{k}}^{\mathrm{diff}}\left(t-t_{k}\right)} \Pi_{i_{k}} x\left(t_{k}-\right), \quad t \in\left[t_{k}, t_{k+1}\right) .
\end{aligned}
$$

Let $t_{0}<t_{1}<t_{2}<\ldots$ be the switching times of $\sigma$ and $i_{k} \in\{1,2, \ldots, M\}$ the value of $\sigma$ on $\left[t_{k}, t_{k+1}\right)$. Then for $t \in\left[t_{k}, t_{k+1}\right)$ the solution of the switched DAE (1) can be represented therefore as

$$
\begin{array}{r}
x(t)=e^{A_{i_{k}}^{\text {diff }}\left(t-t_{k}\right)} \Pi_{i_{k}} e^{A_{i_{k-1}}^{\text {diff }}\left(t_{k}-t_{k-1}\right)} \Pi_{i_{k-1}} \ldots \\
e^{A_{i_{1}}^{\text {diff }}\left(t_{2}-t_{1}\right)} \Pi_{i_{1}} e^{A_{i_{0}}^{\text {diff }}\left(t_{1}-t_{0}\right)} \Pi_{i_{0}} x\left(t_{0}-\right) .
\end{array}
$$

Remark 1 (Impulsive solution): If the impulse-freeness condition (18) is not fulfilled then the switched DAE (1) with regular matrix pairs $\left(E_{i}, A_{i}\right)$ has unique solutions within a suitable distributional solution framework [12]. Each distributional solution $x$ can then be written as $x=x_{f}+x_{i}$ where $x_{f}$ corresponds to a piecewise-smooth function and $x_{i}$ contains the impulsive terms (which only occur at the switching times). The solution formula (21) still holds for $x_{f}$, so the forthcoming results can also be applied to switched DAEs which do not satisfy the impulse-freeness condition (18) provided one is only interested in the non-impulsive part $x_{f}$ of the solution. In fact, the impulses in $x$ are proportional to the jumps and since the averaging model results in smooth solutions it is to be expected that the impulsive part converges to zero as the non-impulsive part converges to the averaged solution; however, we haven't studied this aspect in detail yet.

\section{AVERAGING FOR SWITCHED DAES}

Consider the switched DAE initial value problem defined on the time interval $[0, \infty)$ :

$$
\begin{aligned}
E_{\sigma} \dot{x} & =A_{\sigma} x \\
x(0-) & =x_{0} .
\end{aligned}
$$

Assume that $\sigma:[0, \infty) \rightarrow\{1,2\}$ is periodic with period $p$ and given by

$$
\sigma(t)= \begin{cases}1, & t \in[k p,(k+d) p), k \in \mathbb{N} \\ 2, & t \in[(k+d) p,(k+1) p), k \in \mathbb{N}\end{cases}
$$

where $d \in(0,1)$ is the duty cycle of mode one.

To define an averaged model for this class of systems a naive generalization from the ODE case to the DAE case is not working, as shown in the following simple example.

Example 1: Let

$$
\begin{aligned}
& \left(E_{1}, A_{1}\right)=\left(\left[\begin{array}{ll}
0 & 1 \\
0 & 0
\end{array}\right],\left[\begin{array}{cc}
0 & -1 \\
1 & -1
\end{array}\right]\right), \\
& \left(E_{2}, A_{2}\right)=\left(\left[\begin{array}{ll}
0 & 0 \\
0 & 1
\end{array}\right],\left[\begin{array}{cc}
1 & 0 \\
0 & -3
\end{array}\right]\right),
\end{aligned}
$$

and $\sigma$ be periodic with period $p>0$ and $d=0.75$. The matrix pairs $\left(T_{1}, S_{1}\right)$ and $\left(T_{2}, S_{2}\right)$ corresponding to the quasi Weierstrass form (10) are

$$
\begin{aligned}
& \left(T_{1}, S_{1}\right)=\left(\left[\begin{array}{ll}
1 & 1 \\
1 & 0
\end{array}\right],\left[\begin{array}{ll}
1 & 0 \\
0 & 1
\end{array}\right]\right), \\
& \left(T_{2}, S_{2}\right)=\left(\left[\begin{array}{ll}
0 & 1 \\
1 & 0
\end{array}\right],\left[\begin{array}{ll}
0 & 1 \\
1 & 0
\end{array}\right]\right),
\end{aligned}
$$

the consistency projectors are

$$
\Pi_{1}=\left[\begin{array}{ll}
0 & 1 \\
0 & 1
\end{array}\right], \quad \Pi_{2}=\left[\begin{array}{ll}
0 & 0 \\
0 & 1
\end{array}\right],
$$

and the corresponding flow matrices are

$$
A_{1}^{\text {diff }}=\left[\begin{array}{cc}
0 & -1 \\
0 & -1
\end{array}\right], \quad A_{2}^{\text {diff }}=\left[\begin{array}{cc}
0 & 0 \\
0 & -3
\end{array}\right] .
$$

Solutions for the corresponding switched DAE are shown in Figure 1. It can be seen, that the solutions jump back and forth between the two consistency spaces; clearly, increasing the switching frequency does not lead to a single smooth trajectory. Hence, convergence to an average model is not possible. 


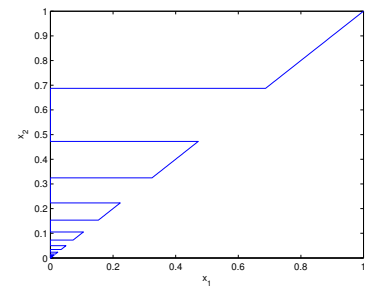

(a)

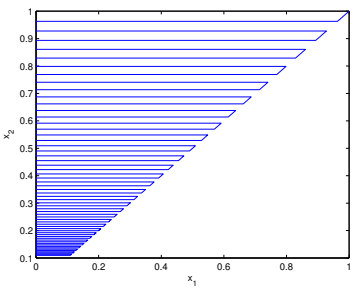

(b)
Fig. 1. State-space solution of Example (1): (a) $p=0.5 \mathrm{~s}$, (b) $p=0.05 \mathrm{~s}$.

The previous example showed a major difference to the switched ODE case where under no further assumptions convergence to an average model appears. For switched DAEs it is intuitively clear, that in case of convergence to trajectories of an averaged model, these trajectories must evolve within the intersection of the consistency spaces otherwise the jumps will not converge to zero as the frequency increases. Furthermore, at least one consistency projector must jump into the intersection, otherwise the limit cannot be within the intersection. It turns out that the crucial assumption is commutativity of the consistency projectors:

$$
\Pi_{1} \Pi_{2}=\Pi_{2} \Pi_{1} \text {. }
$$

By Lemma (1) this condition already implies that the image of $\Pi_{1}$ (applied to the consistency space $i m \Pi_{2}$ ) is contained within the intersection of the consistency spaces, in fact

$$
\Pi_{\cap}:=\Pi_{1} \Pi_{2} \text { fulfills } \quad \operatorname{im} \Pi_{\cap}=\operatorname{im} \Pi_{1} \cap \operatorname{im} \Pi_{2} .
$$

We are now ready to state our main result:

Theorem 2: Consider the switched DAE system (22) with two modes satisfying the following assumptions

(i) the switching signal $\sigma$ is periodic of period $p$ and given by (23) with $d \in(0,1)$;

(ii) the matrix pairs $\left(E_{i}, A_{i}\right), i=1,2$, are regular with corresponding consistency projectors $\Pi_{i}$ and flow matrices $A_{i}^{\text {diff; }}$

(iii) the consistency projectors commute, i.e. (24) holds; in particular $\Pi_{\cap}$ fulfills (25);

then for any given $\bar{t}>0$ and $x_{0} \in \mathbb{R}^{n}$ the following holds

$$
\left\|x(t)-x_{\mathrm{av}}(t)\right\|=O(p), \quad \forall t \in(0, \bar{t}],
$$

where $x_{\mathrm{av}}$ is the solution of

$$
\dot{x}_{\mathrm{av}}=\Pi_{\cap} A_{\mathrm{av}}^{\mathrm{diff}} \Pi_{\cap} x_{\mathrm{av}}, \quad x_{\mathrm{av}}(0)=\Pi_{\cap} x_{0},
$$

and $A_{\mathrm{av}}^{\mathrm{diff}}:=A_{1}^{\mathrm{diff}} d+A_{2}^{\mathrm{diff}}(1-d)$.

Proof: Considering the arbitrary but fixed time instant $t^{*} \in(0, \bar{t}]$. Choose $k \in \mathbb{N}$ such that $t^{*}=k p+\tau$ for $\tau \in[0, p)$. Note that $t^{*}>0$ implies that $k>0$ for sufficiently small $p$. The solution of the switched DAE can then be written as

$$
x\left(t^{*}\right)=\tilde{M}(\tau) \Pi_{1}\left(e^{A_{2}^{\mathrm{diff}}(1-d) p} \Pi_{2} e^{A_{1}^{\mathrm{diff}} d p} \Pi_{1}\right)^{k} x_{0},
$$

where

$$
\tilde{M}(\tau)=\left\{\begin{array}{lll}
e^{A_{1}^{\mathrm{diff}} \tau} & \text { if } & 0 \leqslant \tau<d p \\
e^{A_{2}^{\text {diff }}(\tau-d p)} \Pi_{2} e^{A_{1}^{\mathrm{diff}} d p} & \text { if } & d p \leqslant \tau<p .
\end{array}\right.
$$

Invoking (16) as well as $\Pi_{\cap}^{2}=\Pi_{\cap}$, we can rewrite (28) as

$$
\begin{aligned}
x\left(t^{*}\right) & =\tilde{M}(\tau) \Pi_{1}\left(\Pi_{2} e^{A_{2}^{\text {diff }}(1-d) p} e^{A_{1}^{\text {diff }} d p} \Pi_{1}\right)^{k} x_{0} \\
& =M(\tau)\left(\Pi_{\cap} e^{A_{2}^{\text {diff }}(1-d) p} e^{A_{1}^{\text {diff }} d p} \Pi_{\cap}\right)^{k-1} N(p) x_{0},
\end{aligned}
$$

with

$$
M(\tau):=\tilde{M}(\tau) \Pi_{\cap}=\Pi_{\cap}+O(p)
$$

and

$$
N(p):=\Pi_{\cap} e^{A_{2}^{\text {diff }}(1-d) p} e^{A_{1}^{\text {diff }} d p} \Pi_{1}=\Pi_{\cap}+O(p),
$$

where we used the constant approximation of the exponential(s) at zero and the fact that $\Pi_{\cap} \Pi_{1}=\Pi_{\cap}=\Pi_{2} \Pi_{\cap}$.

By considering the linear approximations of the exponentials in (29) we obtain

$$
\begin{aligned}
x\left(t^{*}\right)= & M(\tau)\left[\Pi_{\cap}\left(I+A_{2}^{\mathrm{diff}}(1-d) p+O\left(p^{2}\right)\right)\right. \\
& \left.\left(I+A_{1}^{\mathrm{diff}} d p+O\left(p^{2}\right)\right) \Pi_{\cap}\right]^{k-1} N(p) x_{0} \\
= & M(\tau)\left(\Pi_{\cap}+\Pi_{\cap} A_{\mathrm{av}}^{\mathrm{diff}} \Pi_{\cap} p+O\left(p^{2}\right)\right)^{k-1} N(p) x_{0} .
\end{aligned}
$$

Note that by construction $O\left(p^{2}\right)=\Pi_{\cap} O\left(p^{2}\right) \Pi_{\cap}$. We will now show that

$$
\begin{aligned}
\left(\Pi_{\cap}+\Pi_{\cap} A_{\mathrm{av}}^{\mathrm{diff}}\right. & \left.\Pi_{\cap} p+O\left(p^{2}\right)\right)^{k-1} \\
& =\left(\Pi_{\cap}+\Pi_{\cap} A_{\mathrm{av}}^{\mathrm{diff}} \Pi_{\cap} p\right)^{k-1}+O(p) .
\end{aligned}
$$

In fact, (33) is similar to the statement in numerics, that the Euler method has global convergence of order one, hence we only sketch the proof idea ${ }^{1}$ here. First consider the map $f: M \mapsto M^{k-1}$ for some square matrices $M$. The derivative of $f$ satisfies $\left\|f^{\prime}(M)\right\| \leqslant(k-1)\|M\|^{k-2}$ for some compatible norms. Now the mean value theorem (see e.g. [19, Thm. VII.3.9]) yields:

$$
\begin{aligned}
\|(M+\Delta)^{k-1}- & M^{k-1}\left\|\leqslant \sup _{\|\bar{\Delta}\| \leqslant\|\Delta\|}\right\| f^{\prime}(M+\bar{\Delta})\|\| \Delta \| \\
& \leqslant(k-1)(\|M\|+\|\Delta\|)^{k-2}\|\Delta\| .
\end{aligned}
$$

Now, invoking $O\left(p^{2}\right)=\Pi_{\cap} O\left(p^{2}\right) \Pi_{\cap}, \Pi_{\cap}^{k}=\Pi_{\cap}$ and (34) for $M=I+A_{\mathrm{av}}^{\text {diff }}$,

$$
\begin{aligned}
& \left\|\left(\Pi_{\cap}+\Pi_{\cap} A_{\mathrm{av}}^{\text {diff }} \Pi_{\cap} p+O\left(p^{2}\right)\right)^{k-1}-\left(\Pi_{\cap}+\Pi_{\cap} A_{\mathrm{av}}^{\text {diff }} \Pi_{\cap} p\right)^{k-1}\right\| \\
& \leqslant\left\|\Pi_{\cap}\right\|^{2}\left\|\left(I+A_{\mathrm{av}}^{\text {diff }} p+O\left(p^{2}\right)\right)^{k-1}-\left(I+A_{\mathrm{av}}^{\text {diff }} p\right)^{k-1}\right\| \\
& \leqslant(k-1)\left(1+\left\|A_{\mathrm{av}}^{\text {diff }}\right\| p+C_{1} p^{2}\right)^{k-2} C_{2} p^{2},
\end{aligned}
$$

where $C_{1}>0$ is some suitable constant independent of $p$ and $k$ and $C_{2}=\left\|\Pi_{\cap}\right\|^{2} C_{1}$. We can assume that $p \leqslant 1$, hence for $C_{3}:=\left\|A_{\mathrm{av}}^{\text {diff }}\right\|+C_{1}$ we obtain

$$
\begin{aligned}
\left(1+\left\|A_{\mathrm{av}}^{\mathrm{diff}}\right\| p+C_{1} p^{2}\right)^{k-2} & \leqslant\left(1+C_{3} p\right)^{k-2} \\
& \leqslant\left(1+\frac{C_{3} t^{*}}{k-2}\right)^{k-2} \leqslant e^{C_{3} t^{*}},
\end{aligned}
$$

${ }^{1}$ We thank Tobias Damm for pointing us in the right direction. 
where the bound $e^{C_{3} t^{*}}$ is independent of $p$ and $k$. Finally invoking $(k-1) e^{C_{3} t^{*}} C_{2} p^{2}=O(p)$ implies (33).

Altogether, by using (30), (31) and (33), equation (32) becomes

$$
\begin{aligned}
x\left(t^{*}\right)= & \left(\Pi_{\cap}+O(p)\right)\left[\left(\Pi_{\cap}+\Pi_{\cap} A_{\mathrm{av}}^{\mathrm{diff}} \Pi_{\cap} p\right)^{k-1} x_{0}\right. \\
& +O(p)]\left(\Pi_{\cap}+O(p)\right) \\
& =\Pi_{\cap}\left(\Pi_{\cap}+\Pi_{\cap} A_{\mathrm{av}}^{\mathrm{diff}} \Pi_{\cap} p\right)^{k-1} \Pi_{\cap} x_{0}+O(p) \\
& \triangleq\left(\Pi_{\cap}+\Pi_{\cap} A_{\mathrm{av}}^{\mathrm{diff}} \Pi_{\cap} p\right)^{k-1} x_{0}+O(p),
\end{aligned}
$$

where for $\diamond$ we use that $\Pi_{\cap}^{k-1}=\Pi_{\cap}$.

Consider now the solution of the averaged model (27)

$$
x_{\mathrm{av}}\left(t^{*}\right)=e^{\Pi_{\cap} A_{\mathrm{av}}^{\mathrm{diff}} \Pi_{\cap} t} \Pi_{\cap} x_{0} .
$$

Note that, for all $s \in \mathbb{R}$,

$$
e^{\Pi_{\cap} A_{\mathrm{av}}^{\mathrm{diff}} \Pi_{\cap} s}=\Pi_{\cap} e^{\Pi_{\cap} A_{\mathrm{av}}^{\mathrm{diff}} \Pi_{\cap} s} \Pi_{\cap},
$$

hence (36) can be written as

$$
\begin{aligned}
x_{\mathrm{av}}\left(t^{*}\right) & =M_{\mathrm{av}}(\tau)\left(\Pi_{\cap} e^{\Pi_{\cap} A_{\mathrm{av}}^{\mathrm{diff}} \Pi_{\cap} p} \Pi_{\cap}\right)^{k} x_{0} \\
& =M_{\mathrm{av}}(\tau)\left(\Pi_{\cap} e^{\Pi_{\cap} A_{\mathrm{av}}^{\mathrm{diff}} \Pi_{\cap} p} \Pi_{\cap}\right)^{k-1} N_{\mathrm{av}}(p) x_{0}
\end{aligned}
$$

with

$$
M_{\mathrm{av}}(\tau)=e^{\Pi_{\cap} A_{\mathrm{av}}^{\mathrm{diff}} \Pi_{\cap} \tau} \Pi_{\cap}=\Pi_{\cap}+O(p),
$$

and

$$
N_{\mathrm{av}}(p)=\Pi_{\cap} e^{\Pi_{\cap} A_{\mathrm{av}}^{\mathrm{diff}} \Pi_{\cap} \tau} \Pi_{\cap}=\Pi_{\cap}+O(p) .
$$

Using again the linear approximation of (37) we obtain

$$
x_{\mathrm{av}}\left(t^{*}\right)=M_{\mathrm{av}}(\tau)\left(\Pi_{\cap}+\Pi_{\cap} A_{\mathrm{av}}^{\mathrm{diff}} \Pi_{\cap} p+O\left(p^{2}\right)\right)^{k-1} N_{\mathrm{av}}(p) x_{0} .
$$

Due to (33), and by using the constant approximation of the exponentials (38) and (39), we can write

$$
x_{\mathrm{av}}\left(t^{*}\right)=\left(\Pi_{\cap}+\Pi_{\cap} A_{\mathrm{av}}^{\mathrm{diff}} \Pi_{\cap} p\right)^{k-1} x_{0}+O(p) .
$$

Comparing now (35) with (40) we obtain

$$
\left\|x\left(t^{*}\right)-x_{\mathrm{av}}\left(t^{*}\right)\right\|=O(p) .
$$

Remark 2: Note that if the duty ratio $d$ is equal to zero (or to one), i.e. the switched DAE reduces to one subsystem, then the convergence to the averaged model is not guaranteed because the trajectory jumps only one time at the beginning and the jump might not be into the intersection of the consistency spaces.

Remark 3: A careful analysis of the proof shows that the constant in the $O(p)$ term in (26) depends exponentially on the end time $t^{*}$ and linearly on the other parameters (e.g. initial value $x_{0}$, magnitudes of the matrices $A_{i}^{\text {diff }}$ and $\Pi_{i}$ ). Note furthermore that (26) only holds for sufficiently small $p$ where "sufficiently" actually depends on $t$ : For $t>0$ the switching period must fulfill $p<t$, because otherwise the inconsistent initial value doesn't jump at least once to the intersection of the consistency spaces and the error could be arbitrary large (c.f. Figures 3 and 4).

Remark 4 (Commutativity and $M>2$ ): A straight forward generalization of the proof idea to the case $M>2$ does not work because in order to obtain the expression (29) the fact that $M=2$ is crucial, because in this case the projectors $\Pi_{1}$ and $\Pi_{2}$ can be moved to the "outside" and are then combined to the single projector $\Pi_{\cap}=\Pi_{1} \Pi_{2}$. For $M>2$ there will be at least one projector which cannot be moved to the outside because then it has to commute with one of the other $A^{\text {diff }}$-matrices. The latter is fulfilled when it is assumed that all $A^{\text {diff }}$ matrices commute [20] which in turn also implies commutativity of the consistency projectors. In that case a generalization of our result is possible, but it seems that these assumptions are too strong in general.

\section{Circuit example}

Consider the simple switched circuit as shown in Figure 2.

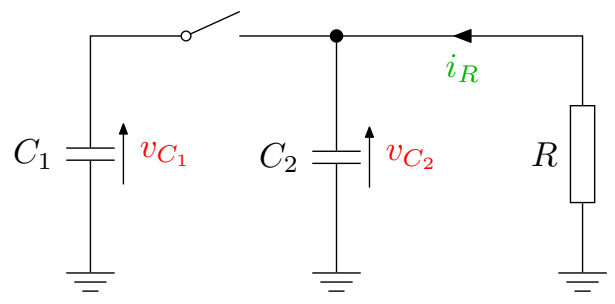

Fig. 2. A simple circuit composed of two parallel capacitors, a switch and a resistor. Note that closing the switch will result in jumps in the voltages; in particular, it is not possible to model this circuit with usual switched ODEs.

The governing equations for the open switch are as follows:

$$
C_{1} \dot{v}_{C_{1}}=0, \quad C_{2} \dot{v}_{C_{2}}=-i_{R}
$$

for the closed switch:

$$
C_{1} \dot{v}_{C_{1}}+C_{2} \dot{v}_{C_{2}}=-i_{R}, \quad 0=v_{C_{1}}-v_{C_{2}},
$$

and $0=v_{C_{2}}-R i_{R}$ in both cases. With $x=\left(v_{C_{1}}, v_{C_{2}}, i_{R}\right)^{\top}$, this results in a switched DAE given by

$$
\begin{aligned}
& \left(E_{1}, A_{1}\right)=\left(\left[\begin{array}{ccc}
0 & 0 & 0 \\
C_{1} & 0 & 0 \\
0 & C_{2} & 0
\end{array}\right],\left[\begin{array}{ccc}
0 & -1 & -R \\
0 & 0 & 0 \\
0 & 0 & -1
\end{array}\right]\right) \\
& \left(E_{2}, A_{2}\right)=\left(\left[\begin{array}{ccc}
0 & 0 & 0 \\
C_{1} & C_{2} & 0 \\
0 & 0 & 0
\end{array}\right],\left[\begin{array}{ccc}
0 & 1 & -R \\
0 & 0 & 1 \\
1 & -1 & 0
\end{array}\right]\right)
\end{aligned}
$$

The corresponding consistency projectors are given by:

$$
\Pi_{1}=\left[\begin{array}{lll}
1 & 0 & 0 \\
0 & 1 & 0 \\
0 & \frac{1}{R} & 0
\end{array}\right], \quad \Pi_{2}=\frac{1}{C_{1}+C_{2}}\left[\begin{array}{ccc}
C_{1} & C_{2} & 0 \\
C_{1} & C_{2} & 0 \\
\frac{C_{1}}{R} & \frac{C_{2}}{R} & 0
\end{array}\right] .
$$

It is easy to check that both projectors commute:

$$
\Pi_{\cap}:=\Pi_{1} \Pi_{2}=\Pi_{2}=\Pi_{2} \Pi_{1},
$$

hence we can define the averaged system as follows:

$$
\dot{x}=\Pi_{\cap} A_{\mathrm{av}}^{\mathrm{diff}} \Pi_{\cap} x=\frac{-1}{R\left(C_{1}+C_{2}\right)^{2}}\left[\begin{array}{ccc}
C_{1} & C_{2} & 0 \\
C_{1} & C_{2} & 0 \\
\frac{C_{1}}{R} & \frac{C_{2}}{R} & 0
\end{array}\right] .
$$

Note that for this example the averaged systems does not depend on the duty cycle $d \in(0,1)$ (but $A_{\mathrm{av}}^{\text {diff }}$ does). The solution of the corresponding switched DAEs and that of the averaged system (41) are shown in Fig 3, with a switching period $p=0.1 \mathrm{~s}$. By decreasing the switching period the solution of the switched DAE and that of the averaged model become close to each other as shown in Fig. 4, where a switching period $p=0.02 \mathrm{~s}$ is chosen. 


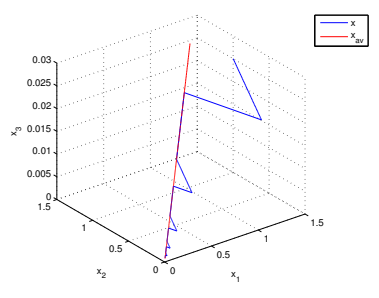

(a)

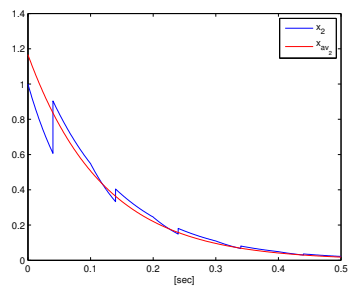

(c)

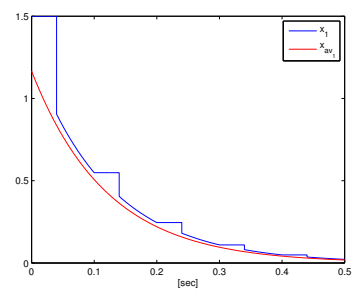

(b)

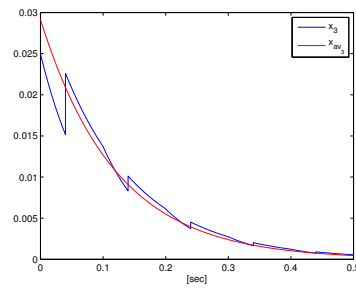

(d)
Fig. 3. Simulation results for the circuit as shown in Figure 2, with $p=$ $0.1 \mathrm{~s}$ : (a) state-space solutions, (b) time evolution of $x_{1}$, (c) time evolution of $x_{2}$, (d) time evolution of $x_{3}$.

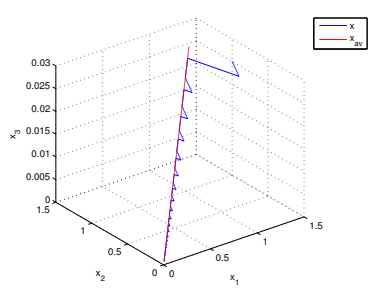

(a)

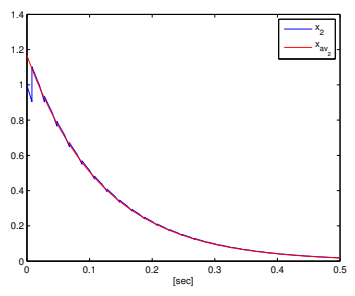

(c)

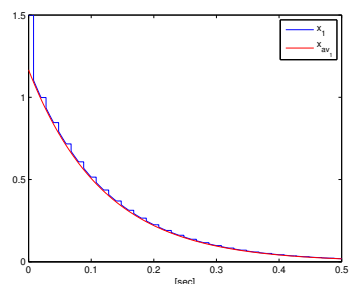

(b)

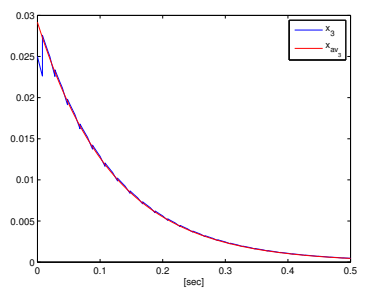

(d)
Fig. 4. Simulation results for the circuit as shown in Figure 2, with $p=$ $0.02 \mathrm{~s}$ : (a) state-space solutions, (b) time evolution of $x_{1}$, (c) time evolution of $x_{2}$, (d) time evolution of $x_{3}$.

\section{CONCLUSIONS}

This paper studied the averaging method for linear switched differential algebraic equations (DAEs). Via a simple example we showed that a trivial generalization of the ODE case is not possible due to the presence of jumps. By assuming that the consistency projectors commute we define an averaged model in the case of the homogeneous linear switched DAEs with the switching signal that periodically switches between two modes. The main result (Theorem 2) says that the error between the solution of the switched DAEs and that of the corresponding averaged model is of the order of the switching period. The work reported here is just an initial step in the investigation of averaging techniques for switched DAEs and opens interesting lines of research. For instance it could be of interest to define an averaged model considering switched DAEs with more than two modes, or for solution with impulses. Other directions of future research could be to investigate averaging for switched DAEs with state-dependent switching functions. Also, the possible usefulness of the averaged system for the stability analysis is an interesting challenge.

\section{REFERENCES}

[1] H. Khalil, Nonlinear Systems, 3rd ed. Upper Saddle River, New Jersey: Prentice Hall, 2002.

[2] S. Almér and U. Jönsson, "Harmonic analysis of pulse-width modluated systems," Automatica, vol. 45, no. 4, pp. 851-862, 2009.

[3] M. Porfiri, D. G. Roberson, and D. J. Stilwell, "Fast switching analysis of linear switched systems using exponential splitting," SIAM Journal of Control Optimization, vol. 47, no. 5, pp. 2582-2597, 2008.

[4] A. Teel, L. Moreau, and D. Nešić, "Input-to-state set stability of pulse width modulated systems with disturbances," Systems and Control Letters, vol. 51, no. 1, pp. 23-32, 2004.

[5] L. Iannelli, K. Johansson, U. Jönsson, and F. Vasca, "Averaging of nonsmooth systems using dither," Automatica, vol. 42, no. 4, pp. 669676, 2006.

[6] — "Subtleties in the averaging of a class of hybrid systems with applications to power converters," Control Engineering Practice, vol. 18, no. 8, pp. 961-975, 2008.

[7] W. Wang and D. Nešić, "Input-to-state set stability and averaging of linear fast switching systems," IEEE Transactions on Automatic Control, vol. 55, no. 5, pp. 1274-1279, 2010.

[8] A. Teel and D. Nešić, "Averaging theory for a class of hybrid systems," Dynamics of Continuous, Discrete and Impulsive Systems, vol. 17, no. 6, pp. 829-851, 2010.

[9] C. Pedicini, F. Vasca, L. Iannelli, and U. Jonsson, "An overview on averaging for pulse-modulated switched systems," in Proc. 50th IEEE Conf. Decis. Control and European Control Conf. ECC 2011, Orlando, USA, dec. 2011, pp. $1860-1865$.

[10] C. Pedicini, L. Iannelli, and F. Vasca, "The averaging method for control design and stability analysis of practical switched systems," in Proc. 2012 IEEE Multi-Conf. on Systems and Control(MSC), oct. 2012, pp. $1285-1290$

[11] D. Liberzon and S. Trenn, "Switched nonlinear differential algebraic equations: Solution theory, Lyapunov functions, and stability," Automatica, vol. 48, no. 5, pp. $954-963,2012$.

[12] S. Trenn, "Switched differential algebraic equations," in Dynamics and Control of Switched Electronic Systems - Advanced Perspectives for Modeling, Simulation and Control of Power Converters, F. Vasca and L. Iannelli, Eds. London: Springer, 2012, ch. 6, pp. 189-216.

[13] A. D. Domínguez-García and S. Trenn, "Detection of impulsive effects in switched DAEs with applications to power electronics reliability analysis," in Proc. 49th IEEE Conf. Decis. Control, Atlanta, USA, 2010, pp. 5662-5667.

[14] J. Ezzine and A. H. Haddad, "Error bounds in the averaging of hybrid systems," IEEE Transactions on Automatic Control, vol. 34, no. 11, pp. 1188-1192, 1989.

[15] K. Weierstraß, "Zur Theorie der bilinearen und quadratischen Formen," Berl. Monatsb., pp. 310-338, 1868.

[16] T. Berger, A. Ilchmann, and S. Trenn, "The quasi-Weierstrass form for regular matrix pencils," Linear Algebra and its Applications, vol. 436, no. 10, pp. $4052-4069,2012$.

[17] K.-T. Wong, "The eigenvalue problem $\lambda T x+S x$," J. Diff. Eqns., vol. 16, pp. 270-280, 1974.

[18] A. Tanwani and S. Trenn, "On observability of switched differentialalgebraic equations," in Proc. 49th IEEE Conf. Decis. Control, Atlanta, USA, dec. 2010, pp. $5656-5661$.

[19] H. Amann and J. Escher, Analysis II, 1st ed. Basel Boston Berlin: Birkhäuser, 2008.

[20] D. Liberzon, S. Trenn, and F. R. Wirth, "Commutativity and asymptotic stability for linear switched DAEs," in Proc. 50th IEEE Conf. Decis. Control and European Control Conf. ECC 2011, Orlando, USA, 2011, pp. 417-422. 